\title{
A PROBLEM IN TWO-DIMENSIONAL INTEGRATION
}

\section{RALPH HENSTOCK}

(Received 12 May 1982)

Communicated by $\mathbf{G}$. Brown

\begin{abstract}
If two functions of a real variable are integrable over two intervals, say of $t, \tau$, respectively, then the product of the two functions should be integrable over the rectangular product of the two intervals of $t$ and $\tau$. For the Lebesgue integral, definable using non-negative functions alone, the proof is easy. For non-absolute integrals such as the Perron, Cesàro-Perron, and Marcinkiewicz-Zygmund integrals we have difficulties since the functions cannot be assumed non-negative. But the present paper gives a proof.
\end{abstract}

1980 Mathematics subject classification (Amer. Math. Soc.): 26 A 39.

A problem posed recently by Washek Pfeffer is formulated as Theorem 1 below.

Theorem 1. If $f(t), \varphi(\tau)$ are Perron integrable to $F(a, b), \Phi(\alpha, \beta)$ over the intervals $[a, b],[\alpha, \beta]$ of the real line, respectively, then $f(t) \varphi(\tau)$ is Perron integrable over $[a, b] \times[\alpha, \beta]$ to $F(a, b) \Phi(\alpha, \beta)$, that is,

$$
\int_{[a, b] \times[\alpha, \beta]} f(t) \varphi(\tau) d(t, \tau)=\int_{[a, b]} f(t) d t \int_{[\alpha, \beta]} \varphi(\tau) d \tau .
$$

This is easy for Lebesgue integration, and $F$ is a Lebesgue integral when $f \geqslant 0$. [26], page 63, Theorem 7, is more general, but the assumption of bounded variation shows that we are again dealing with absolute integrals. The problem ought to be easy even when the integrals are not absolute, but the changing of sign of $f$ and $\varphi$ causes difficulties. A quick proof notes several integral equivalences, namely, the Perron integral is equivalent to the Ward integral, to the variational integral, and to the Riemann-complete integral, a form of the generalized Riemann integral, see $[49,21,25]$. Then [28], page 83 , Theorem 11, with the 
$h_{j}$ there of generalized bounded variation, the remarks just after the proof of the theorem, and $f\left(x_{1}, x_{2}\right)=1$ everywhere, finish the proof. But, because of the integral equivalences and the remarks in [28] that are too brief, this is not a clear lucid proof, so the present paper gives a proof of the Perron integral result using variational integration. The author was sorely tempted to give the theorem in a much more general setting, such as that of $W$. Pfeffer $[40,41]$ or in division spaces. But in the interests of readability the simple case for a two-dimensional rectangle is given first, and then a similar proof for certain convergence-factor integrals on the two-dimensional rectangle. This last result seems to be the first theorem for product spaces using convergence-factor integrals, and it includes the straightforward Perron result, but the Perron result is given so as to illuminate and provide a pattern for the more difficult part, and to start an inductive chain. The reason for using convergence-factor integrals in mathematics is that for some everywhere convergent trigonometric series, the sum

$$
f(t)=\frac{1}{2} a_{0}+\sum_{n=1}^{\infty}\left(a_{n} \cos n t+b_{n} \sin n t\right)
$$

is not Denjoy integrable. Thus more general integrals were introduced using generalized derivatives. For fixed $r \geqslant 1$, J. C. Burkill $[7,8,9]$ used right-hand derivates

$$
\text { (3) } \lim _{v \rightarrow u^{+}}(S) \int_{u}^{v} n_{+}(u, v, t)(F(t)-F(u)) d t /(S) \int_{u}^{v} n_{+}(u, v, t)(t-u) d t
$$

of $F$ with $n_{+}(u, v, t)=r(v-t)^{r-1} /(v-u)^{r},(S)$ being Çesàro-Perron integration of order $r-1$ when $r \geqslant 2$ and Perron integration when $1 \leqslant r<2$; and left-hand derivates of $F$

$$
\text { (4) } \lim _{u \rightarrow v_{-}}(S) \int_{u}^{v} n_{-}(u, v, t)(F(v)-F(t)) d t /(S) \int_{u}^{v} n_{-}(u, v, t)(v-t) d t
$$

with the same $(S)$ and $n_{-}(u, v, t)=r(t-u)^{t-1} /(v-u)^{r}$, to define Çesàro-Perron integrals of order $r$, giving an inductive chain of integrals. See also L. S. Bosanquet [2], W. L. C. Sargent [43], and S. Verblunsky [47]. H. W. Ellis [16] started from the general Denjoy integral and defined a scale of mean-continuous integrals more general than the scale of Çesàro-Perron integrals, see P. S. Bullen and C. M. Lee [4], page 495, 6.2. J. Marcinkiewicz and A. Zygmund [34] used Borel derivates with Perron integration $(S)$,

$$
\begin{array}{r}
\lim _{h \rightarrow 0+}(S) \int_{u-h}^{u+h} n(u-h, u+h, t)(F(t)-F(u)) d t /(S) \int_{u-h}^{u+h} n(u-h, \\
u+h, t)(t-u) d t
\end{array}
$$


where $n(u-h, u+h, t)=\frac{1}{2}(t-u)^{-1}(t \neq u), n(u-h, u+h, u)=0$, for their $T$ - or $M Z$-integral. For Burkill's symmetric Çesàro-Perron (SCP) integral [10], $n(u-h, u+h, t)$ is $\operatorname{sgn}(t-u),+1$ if $t>u,-1$ if $t<u$. In both cases of (5) the constant $F(u)$ in the integrals cancels. R. L. Jeffery and D. S. Miller [32] gave a general definition with (3), (4), positive functions $n_{+}, n_{-}$, and $(S)$ some suitably defined integration process. [32], p. 128, assumed an unnecessary limit, namely, for Lebesgue integration,

$$
\frac{1}{c}=\lim _{v \rightarrow u+} \int_{u}^{v} n_{+}(u, v, t)(t-u) d t /\left\{(v-u) \int_{u}^{v} n_{+}(u, v, t) d t\right\},
$$

and similarly for $n_{-}$, but here we disregard this limit and follow [22]. P. S. Bullen and C. M. Lee [4] gave a general theory excluding Stieltjes-type integrals. In [5] they gave a scale of $S C P$-type integrals. Here again, if we wish to generalize Theorem 1 we need $f$ and $\varphi$ that change sign often, for S. Foglio [17] showed that if $f \geqslant 0$ and $g$ is monotone increasing, the $N_{s}$-Perron integral of $f(t)$ with respect to $g(t)$ in [22] is also a Lebesgue-Stieltjes integral. There are other generalized Perron-type integrals, such as integrals between Perron's and the Çesàro-Perron, defined by L. Gordon [19] using for his upper right derivate the greatest lower bound of all constants $a$ for which

$$
\int_{0}^{h}[F(u+t)-F(u)-a t]_{+}^{r} d t=o\left(h^{r+1}\right), \quad[x]_{+} \equiv \max (x, 0),
$$

the integration being Lebesgue's. If no such $a$ exists, the upper right derivate is written $+\infty$. Other integrals use second or higher order derivates, such as the $P^{n}$ - and $\mathscr{P}^{n}$-integrals of R. D. James [30,31], see P. S. Bullen [3], G. E. Cross $[11,12,14,15]$, and S. N. Mukhopadhyay [38], and the Peano derivatives of $S$. Verblunsky [48]. There is also the Abel-Poisson-Perron integral of S. J. Taylor [45]. See G. E. Cross [13].

Convergence-factor integrals may also be needed if one needs path integrals, of functions whose squares are not integrable, for infinite dimensional spaces with Wiener measure, for see H. P. McKean [36], page 31, Problem 1. The oscillation shows the need of some smoothing device.

\section{Perron and variational integrals}

For $\mathbf{R}$ the real line and $[a, b] \subset \mathbf{R}$ a bounded closed interval let $f, M$ be functions from $[a, b]$ to $\mathbf{R}$. Then $M$ is defined a Perron major function of $f$ on $[a, b]$, if

(6) $M$ is continuous on $[a, b]$ with $M(a)=0$,

(7) for $\underline{M}^{\prime}$ the lower derivate of $M, \underline{M}^{\prime} \geqslant f$ almost everywhere on $[a, b]$, 
(8) $\underline{M}^{\prime}>-\infty$ except for a countable set $X$ on $[a, b]$.

This definition is in a sense an amalgam of the worst features of O. Perron [39], H. Bauer [1], H. Hake [20], H. Looman [33], S. Saks [42], pages 186-203, and E. J. McShane [37], page 313, except that McShane used four functions instead of two, and that we use a finite-valued $f$. See D. N. Sarkhel [44]. In Perron integration the integrand is finite almost everywhere, and the exceptional set, in which $f$ is infinite and we can replace $f$ by 0 , can be put with that of (7).

The infimum of $M(b)$ for all Perron major functions $M$ of $f$ on $[a, b]$, is called the upper Perron integral of $f$ on $[a, b]$. An $m:[a, b] \rightarrow R$ is a Perron minor function of $f$ on $[a, b]$, if and only if $-m$ is a Perron major function of $-f$ on $[a, b]$. The lower Perron integral of $f$ on $[a, b]$ is the supremum of $m(b)$ for all such $m$. Strengthening $(7,8)$, we say that $M$ is a strong Perron major function of $f$ on $[a, b]$, if and only if (6) holds with

$$
\underline{M}^{\prime}>f \text { everywhere on }[a, b],
$$

while $m$ is a strong Perron minor function of $f$ on $[a, b]$ if and only if $-m$ is a strong Perron major function of $-f$ on $[a, b]$.

THEOREM 2. The upper and lower Perron integrals of $f$ on $[a, b]$ are unaltered if Perron major and minor functions of $f$ are replaced by strong Perron major and minor functions of $f$, respectively, and in consequence, if $f$ is replaced by an $f^{*}:[a, b] \rightarrow \mathbf{R}$ with $f^{*}=f$ almost everywhere.

Proof. Using (6) on continuity, we remove the sequence $\left(s_{n}\right)$ of points in $X$ of (8). Let

$$
\begin{gathered}
L(t, H)=\sup _{0<|h| \leqslant H}|M(t+h)-M(t)|, \\
L(t, 0)=0, \quad L(t,-H)=-L(t, H) .
\end{gathered}
$$

For each fixed $t, L(t, H)$ is bounded and continuous in $H$ and monotone decreasing to 0 as $H \rightarrow 0+$. Thus, given $\varepsilon>0$, there are an $H_{n}>0$ and $L_{n}(t)$ such that

$$
\begin{gathered}
L\left(s_{n}, H_{n}\right) \leqslant \varepsilon \cdot 2^{-n-1} \quad(n=1,2, \ldots), \\
L_{n}\left(t+s_{n}\right)= \begin{cases}L\left(s_{n}, t\right)-L\left(s_{n},-H_{n}\right) & \left(|t| \leqslant H_{n}\right), \\
L\left(s_{n}, H_{n}\right)-L\left(s_{n},-H_{n}\right) & \left(t \geqslant H_{n}\right), \\
0 & \left(t \leqslant-H_{n}\right) .\end{cases}
\end{gathered}
$$


Then for $0<h \leqslant H_{n}$, using $(10,11,12)$,

$$
\begin{gathered}
L_{n}\left(s_{n}+h\right)-L_{n}\left(s_{n}\right) \geqslant\left|M\left(s_{n}+h\right)-M\left(s_{n}\right)\right|, \\
L_{n}\left(s_{n}\right)-L_{n}\left(s_{n}-h\right) \geqslant\left|M\left(s_{n}\right)-M\left(s_{n}-h\right)\right|, \\
0 \leqslant \sum_{n=1}^{\infty} L_{n} \leqslant \varepsilon, \text { and } M_{1} \equiv M+\sum_{n=1}^{\infty} L_{n}
\end{gathered}
$$

is continuous in $[a, b]$ with $\underline{M}_{1}^{\prime} \geqslant 0$ at each $s_{n}$. As a countable set is of measure zero, $M_{1}$ satisfies (7) while $X$ is empty in (8) for $M_{1}$.

Next we remove the exceptional set $Y$ of measure zero implicit in (7) using a construction of Burkill [10], page 47, Lemma. Given $n$, there is an open set $G_{n} \supseteq Y$ of measure less than $\varepsilon \cdot 4^{-n}$. We define

$$
\begin{aligned}
y_{0 n}(t) & =\left\{\begin{array}{l}
2^{n}\left(t \in G_{n}\right), \\
0\left(t \notin G_{n}\right),
\end{array} \quad y_{n}(t)=\int_{a}^{t} y_{0 n}(t) d t,\right. \\
y(t) & =\sum_{n=1}^{\infty} y_{n}(t), \quad y_{n}(b)<\varepsilon 2^{-n}, \quad y(b)<\varepsilon .
\end{aligned}
$$

Then $y_{n}(t)$ and $y(t)$ are continuous and monotone increasing in $[a, b]$. Putting $M_{2}(t) \equiv M_{1}(t)+y(t)+\varepsilon(t-a), \underline{M}_{1}^{\prime}>-\infty$ gives

$$
\underline{\boldsymbol{M}}_{2}^{\prime}=+\infty(t \in Y), \quad \underline{M}_{2}^{\prime} \geqslant f+\varepsilon>f(t \in[a, b] \backslash Y),
$$

and the continuous $M_{2}$ satisfies (9) everywhere on $[a, b]$. Thus $M_{2}$ is a strong Perron major function of $f$ on $[a, b]$ and Theorem 2 follows from

$$
M(b)<M_{2}(b)<M(b)+\varepsilon(2+b-a) .
$$

By Borel's covering theorem every strong Perron major function of $f$ on $[u, v] \subseteq[a, b]$ is not less than $m$, for each strong Perron minor function $m$ of $f$ on $[u, v]$. Thus, taking $M, m$ for $[a, b]$, we have

$$
M(v)-\boldsymbol{m}(v)-M(u)+m(u) \geqslant 0
$$

and $M-m$ is monotone increasing while the upper integral over $[a, b]$ is not less than the lower integral. Their common value, if equal, is the Perron integral of $f$ on $[a, b]$ and, given $\varepsilon>0$, there are Perron major and minor functions $M, m$ of $f$ with

$$
0 \leqslant M(b)-m(b)<\varepsilon .
$$

Conversely, if (13) is true for all $\varepsilon>0$ and some $M, m$, major and minor functions of $f$ and depending on $\varepsilon$, the Perron integral exists over $[a, b]$. As $M-m$ is monotone increasing the Perron integral exists over $[a, t]$ for each $t$ in $a<t \leqslant b$, say with value $P(t)$, and $M-P, P-m$ are monotone increasing. 
If there is a $P:[a, b] \rightarrow R$ with $P(a)=0$ and the properties that, given $\varepsilon>0$, there are a monotone increasing function $K(t)$ and a $\delta(t)>0$ on $[a, b]$ such that

$$
\begin{gathered}
|K(t+h)-K(t)| \geqslant|P(t+h)-P(t)-f(t) h| \\
\quad(|h| \leqslant \delta(t), t \text { and } t+h \text { in }[a, b]), \\
K(b)-K(a)<\varepsilon,
\end{gathered}
$$

we say that $P$ is the variational integral of $f$ over $[a, b]$. May I emphasize that $f$ is finite-valued.

THEOREM 3. If $M, m$ are strong Perron major and minor functions, respectively, of $f$ on $[a, b]$ then $M-m$ is monotone increasing. Further, if the Perron integral exists over $[a, b]$ it exists, say as $P(t)$, over $[a, t]$, for $a<t \leqslant b$, and if $P(a)=0$ then $P$ is the variational integral of $f$ over $[a, b]$. Conversely, the variational integral is the Perron integral.

Proof. Much of the theorem has already been proved. Let $M$ satisfy (9), so that, for some $\delta_{1}(t)>0$,

$$
\begin{array}{cc}
M(t+h)-M(t)>f(t) h & \left(a \leqslant t<t+h \leqslant b, 0<h \leqslant \delta_{1}(t)\right), \\
M(t)-M(t-h)>f(t) h & \left(a \leqslant t-h<t \leqslant b, 0<h \leqslant \delta_{1}(t)\right) .
\end{array}
$$

If $f$ is Perron integrable to $P(t)$ over $[a, t]$ for each $t$ in $a<t \leqslant b$ then $Q \equiv M-P \geqslant 0$ and is monotone increasing, and we can write (16) as

$$
\begin{aligned}
& Q(t+h)-Q(t)>-\{P(t+h)-P(t)-f(t) h\} \\
&\left(a \leqslant t<t+h \leqslant b, 0<h \leqslant \delta_{1}(t)\right) .
\end{aligned}
$$

For a strong Perron minor function $m$ of $f$ over $[a, b]$, with $q \equiv P-m \geqslant 0$ and monotone increasing, the result corresponding to (18) is

$$
\begin{aligned}
& q(t+h)-q(t)>\{P(t+h)-P(t)-f(t) h\} \\
& \quad\left(a \leqslant t<t+h \leqslant b, 0<h \leqslant \delta_{2}(t)\right) .
\end{aligned}
$$

Replacing $\delta_{1}, \delta_{2}$ by $\delta \equiv \min \left(\delta_{1}, \delta_{2}\right)$, as $Q, q$ are monotone increasing, $(18,19)$ give

$$
\begin{aligned}
Q(t+h)-Q(t)+q(t+h)-q(t) & >|P(t+h)-P(t)-f(t) h| \\
& (a \leqslant t<t+h \leqslant b, 0<h \leqslant \delta(t)) .
\end{aligned}
$$

From (17) and an inequality for $m$, a similar result holds for

$$
a \leqslant t-h<t \leqslant b, \quad 0<h \leqslant \delta(t) .
$$

$Q(a)+q(a)=0, \quad Q(b)+q(b)=M(b)-P(b)+P(b)-m(b)=M(b)-$ $m(b)$, so that by choice of $M, m$, by (13) this is less than $\varepsilon$ and $P$ is the variational 
integral of $f$ over $[a, b]$. Conversely, if for each $\varepsilon>0$ there is a monotone increasing $K$ satisfying $(14,15)$, then by $(14), P+K$ and $P-K$ are Perron major and minor functions, respectively, of $f$ in $[a, b]$, satisfying (9) with $\geqslant$ for $>$, so that by (15), $\boldsymbol{P}$ is the Perron integral of $f$ over $[a, b]$.

We now turn to the original problem, first defining the two-dimensional integral of a function $w: T \equiv[a, b] \times[\alpha, \beta] \rightarrow \mathbf{R}$. By the two-dimensional analogue of Theorem 3 we can use a variational integral definition, and we need the second difference

$$
\Delta^{2} V \equiv \Delta^{2} V(u, t ; \mu, \tau) \equiv V(t, \tau)-V(t, \mu)-V(u, \tau)+V(u, \mu)
$$

(where $V: T \rightarrow \mathbf{R}$ ).

Given $w$, if there is a $W: T \rightarrow \mathbf{R}$ with the properties that, given $\varepsilon>0$, there are a $V: T \rightarrow \mathbf{R}$ with $0 \leqslant \Delta^{2} V<\varepsilon$ for all second differences in $T$, and a $\delta(t, \tau)>0$, such that

$$
\Delta^{2} V(u, t ; \mu, \tau) \geqslant\left|\Delta^{2} W(u, t ; \mu, \tau)-w\left(t^{*}, \tau^{*}\right)(t-u)(\tau-\mu)\right|
$$

for $\left(t^{*}, \tau^{*}\right)$ a vertex of the rectangle $[u, t] \times[\mu, \tau]$ that lies in the circle centre $\left(t^{*}, \tau^{*}\right)$, radius $\delta\left(t^{*}, \tau^{*}\right)$, we say that $\Delta^{2} W(a, b ; \alpha, \beta)$ is the variational integral of $w$ over $T$.

Proof of Theorem 1. Given $\varepsilon>0$ and an integer $j>0$, by Theorems 2,3 there are functions $\delta_{j}(t)>0$ and $K_{j}(t)$ in $[a, b]$ such that

$$
\begin{gathered}
K_{j}(s)-K_{j}(r) \geqslant|f(t)(s-r)-F(r, s)| \quad\left(t=r \text { or } s, 0<s-r \leqslant \delta_{j}(t)\right), \\
K_{j}(b)-K_{j}(a)<\varepsilon \cdot 4^{-j},
\end{gathered}
$$

and there are $\delta_{j}^{*}(\tau)>0, \mathrm{~K}_{j}(\tau)$, relative to $\varphi, \Phi, \alpha, \beta$. Writing

$$
\begin{array}{ll}
K(t) \equiv \sum_{j=1}^{\infty} 2^{j}\left\{K_{j}(t)-K_{j}(a)\right\} & (a \leqslant t \leqslant b), \\
\mathrm{K}(\tau) \equiv \sum_{j=1}^{\infty} 2^{j}\left\{\mathrm{~K}_{j}(\tau)-\mathrm{K}_{j}(\alpha)\right\} & (\alpha \leqslant \tau \leqslant \beta),
\end{array}
$$

then $K(b)<\varepsilon, \mathrm{K}(\beta)<\varepsilon$. If $Z_{0}$ is empty with $Z_{j}$ the set of $(t, \tau)$ in $T$ with $|f(t)| \leqslant 2^{j},|\varphi(\tau)| \leqslant 2^{j}(j=1,2,3, \ldots)$, we put $\delta(t, \tau)=\min \left(\delta_{j}(t), \delta_{j}^{*}(\tau)\right)$ in 
$Z_{j} \backslash Z_{j-1}(j=1,2, \ldots)$ and have for $t=r$ or $s, s>r, \tau=\rho$ or $\sigma, \sigma>\rho$, and

$$
(s-r)^{2}+(\sigma-\rho)^{2} \leqslant \delta^{2}(t, \tau),
$$

$$
\begin{aligned}
f(t) \varphi(\tau)(s-r)(\sigma-\rho)-F(r, s) \Phi(\rho, \sigma) \\
=\{f(t)(s-r)-F(r, s)\} \varphi(\tau)(\sigma-\rho) \\
\quad+f(t)(s-r)\{\varphi(\tau)(\sigma-\rho)-\Phi(\rho, \sigma)\} \\
\quad+\{F(r, s)-f(t)(s-r)\}\{\varphi(\tau)(\sigma-\rho)-\Phi(\rho, \sigma)\}, \\
|f(t) \varphi(\tau)(s-r)(\sigma-\rho)-F(r, s) \Phi(\rho, \sigma)| \\
\leqslant\left\{K_{j}(s)-K_{j}(r)\right\} 2^{j}(\sigma-\rho)+2^{j}(s-r)\left\{\mathrm{K}_{j}(\sigma)-\mathrm{K}_{j}(\rho)\right\} \\
\quad+\left\{K_{j}(s)-K_{j}(r)\right\}\left\{\mathrm{K}_{j}(\sigma)-\mathrm{K}_{j}(\rho)\right\} \\
\leqslant \\
\quad\{K(s)-K(r)\}(\sigma-\rho)+(s-r)\{\mathrm{K}(\sigma)-\mathrm{K}(\rho)\} \\
+\{K(s)-K(r)\}\{\mathrm{K}(\sigma)-\mathrm{K}(\rho)\} .
\end{aligned}
$$

The last expression is non-negative and finitely additive over rectangles, with value

$$
K(b)(\beta-\alpha)+(b-a) \mathrm{K}(\beta)+K(b) \mathrm{K}(\beta)<\varepsilon\{\beta-\alpha+b-a+\varepsilon\}
$$

for $T$. Thus $f \varphi$ is variationally integrable and so Perron integrable over $T$ to $F(a, b) \Phi(\alpha, \beta)$, proving Theorem 1 .

The preceding proofs can easily be given for Perron-Stieltjes integration with strictly increasing $g(t)$ in $[a, b], \gamma(\tau)$ in $[\alpha, \beta]$, on replacing $s-r$ by $\Delta_{1} g(r, s) \equiv$ $g(s)-g(r), \sigma-\rho$ by $\Delta_{2} \gamma(\rho, \sigma) \equiv \gamma(\sigma)-\gamma(\rho)$, with " $g$-almost everywhere" and " $\gamma$-almost everywhere", in appropriate places. When $g, \gamma$ are sometimes constant in intervals or not monotone we replace Perron-Stieltjes integration by Ward [49] integration which is easily shown equivalent to the appropriate variational integration, see [21]. Similar proofs hold for general Denjoy and Burkill's approximate Perron integration [6] as these are equivalent to variational integration over division spaces using intervals and rectangles, see [27,29]. Similarly for $m$-dimensional and $n$-dimensional "rectangles" with $(m+n)$-dimensional Cartesian products, or generalized intervals in product division spaces in which we use finitely additive interval functions instead of the $K, \mathrm{~K}, V$ and their differences. The use of finitely superadditive interval functions causes difficulties illustrated in [26].

\section{Generalized Perron and $N$-variational integration}

In this section we go to convergence-factor integration in intervals and rectangles, following the same pattern of definitions and proofs as in Section 1, but with added complications caused by the convergence-factors. [22] gives necessary 
conditions for integrals of the type using derivatives like $(3,4)$, and [22], page 114 , (27), (28), leads to the following details. Given $a<b$ and each $u$, $v$ in $a \leqslant u<v$ $\leqslant b$ let $N_{s}(u, v, t)(u \leqslant t \leqslant v)$ be monotone increasing in $t$ with

$$
\begin{array}{ll}
N_{s}(u, v, v)-N_{s}(u, v, u)=1 & (s=+,-), \\
N_{+}(u, v, u+)=N_{+}(u, v, u), & N_{-}(u, v, v-)=N_{-}(u, v, v) .
\end{array}
$$

$N_{s}$ is a regular convergence factor for generalized Perron integration in $[a, b]$ using

$$
\begin{aligned}
& I\left(S, N_{s}, F, u, v\right) \equiv(S) \int_{u}^{v} F(t) d_{t} N_{s}(u, v, t) \quad(v>u), \\
& I\left(S, N_{s}, F, u, u\right)=0 \quad(s=+,-),
\end{aligned}
$$

for some Stieltjes integration $(S)$ having all properties proved for $(N)$, if (25) exists for each bounded Baire function $F$ in $[a, b]$ and all $u, v, s$ in $a \leqslant u<v \leqslant b$, $s=+,-$, and if the only functions $F$ with

$$
\delta(t)>0 \text { in }[a, b] \text { and }(u, v) \subseteq(a, b)
$$

and

$$
\begin{array}{ll}
I\left(S, N_{+}, F-F(u), u, v\right) \geqslant 0 & (v-u<\delta(u)), \\
I\left(S, N_{-}, F(v)-F, u, v\right) \geqslant 0, & (v-u<\delta(v)),
\end{array}
$$

are the monotone increasing $F$.

Requirements $(24,27)$ and the additivity in the integrand of the $(S)$-integral give

$$
I\left(S, N_{-}, F, t-h, t\right) \leqslant F(t) \leqslant I\left(S, N_{+}, F, t, t+h\right) \quad(0<h<\delta(t)) .
$$

By [22], page 114, below (28), $(24,25)$ do not give (26), and it is the most difficult condition to arrange. A few special cases are the Çesàro-Perron result of W. L. C. Sargent [43] and those produced by the condition $\nu(x, x+h, t)=$ $\nu(x+h, x, t)$ of R. L. Jeffery and D. S. Miller [32], page 128, called reversibility in [22]. These are generalized in [22], page $116(35,36)$. A result I gave in [18], pages 693-695, Section 1, is corrected and extended in Theorem 4, though the set $B$ is more restricted.

THEOREM 4. Let $B \subseteq[a, b]$ be an intersection of a sequence of open sets $\left(a \mathcal{G}_{\delta}\right.$-set $)$ or in particular a closed set, and let $C=[a, b] \backslash B$.

For each $u, v \in B$ let $N_{s}$ be reversible, $N_{+}(u, v, t)=$ $N_{-}(u, v, t)$ or, more generally, let $F(v) \geqslant F(u)$ when $I\left(S, N_{+}\right.$, $F-F(u), u, w) \geqslant 0, \quad I\left(S, N_{-}, F(v)-F, w, v\right) \geqslant 0$ for all $w$ in $u \leqslant w \leqslant v$. 
For each $t \in C \cap(a, b)$ let there be two infinite sequences of $h$ tending to 0 , one with $h>0$ and one with $h<0$, such that the corresponding $I\left(S, N_{+}, F, t, t+h\right)(h>0)$ and $I\left(S, N_{-}, F, t\right.$ $+h, t)(h<0)$ depend only on the values of $F$ in $B$.

If $F$ satisfies (27) then $F$ is monotone increasing in $[a, b]$.

Proof. By $(24,30), \bar{B} \supseteq C$ so that $\bar{B}=[a, b]$. Let $D$ be the union of all intervals $[v, w] \subseteq[a, b]$ for which $F$ is monotone increasing in $B \cap(v, w)$, so that $[a, b] \backslash D \equiv E$ is a $\mathcal{G}_{\delta}$-set. If $(r, s)$ is a union of the intervals $(v, w)$ then Borel's covering theorem on closed subintervals of $(r, s)$ shows that $F$ is monotone increasing in $B \cap(r, s)$, and so in $[r, s]$ on using $(28,30)$. Thus $F$ is monotone increasing in the component intervals of $D$. If $a \leqslant r<s<t \leqslant b$ and $(r, s)$, $(s, t) \subseteq D$ then $[r, t] \subseteq D$ and $E$ has no isolated points. As $E, B$ are $\mathcal{G}_{\delta}$-sets, so is $E \cap B$. If $E \cap B$ is empty then every interval $(r, s) \subseteq(a, b)$ is a union of the intervals $(v, w)$ and so $F$ is monotone increasing on $[a, b]$. Thus if $E$ is not empty, $E \cap B$ is not empty and for $X_{n}$ the set in (27) with $\delta(t) \geqslant 1 / n$,

$$
E \cap B=\bigcup_{n=1}^{\infty} E \cap B \cap X_{n}
$$

and Baire's density theorem shows that for some integer $n$ and some interval $(r, s) \subseteq(a, b)$ with $s-r<1 / n, Q \equiv E \cap B \cap(r, s)$ is not empty and $Q \cap X_{n}$ is everywhere dense in $Q$. It $t, u \in Q$ with $t<u$ then $t, u \in B$. If $(t, w) \subseteq D$ with $t<w \leqslant u$ then $F(t) \leqslant F(w)$ and we need only prove that $F(w) \leqslant F(u)$. Thus we can assume $t$ unisolated on the right from $E \cap B$ and so from $Q \cap X_{n}$. Similarly we can assume $u$ unisolated on the left from $Q \cap X_{n}$, and there are $t_{1}, u_{1} \in Q \cap X_{n}$ with

$$
\begin{gathered}
t \leqslant t_{1} \leqslant u_{1} \leqslant u, \quad t_{1}-t<\delta(t), \\
u-u_{1}<\delta(u), \quad F(t) \leqslant F\left(t_{1}\right) \leqslant F\left(u_{1}\right) \leqslant F(u),
\end{gathered}
$$

by $(27,29)$, and $F$ is monotone increasing in $E \cap B \cap(r, s)$. As $F$ is monotone increasing in the component intervals of $D$ and by (29), $F$ is monotone increasing in $B \cap(r, s)$ and so in $[r, s]$. Hence $Q$ is empty, giving a contradiction and proving the theorem.

(30) is true if $N_{s}$ is a Lebesgue integral relative to $t$, with $C$ of measure or variation zero. It is also true if $N_{s}$ is a Radon integral relative to a particular strictly increasing $\xi(t)$ with $B$ as the union of sets $P_{j}, P_{1}$ the Cantor set on $[a, b]$, and $P_{j+1}$ as $P_{j}$ with Cantor sets on the intervals of the complement of $\boldsymbol{P}_{j}$ $(j=1,2,3, \ldots)$ so that the Lebesgue measure of $[a, b] \backslash B$ is $b-a$. By Baire's density theorem, as each $P_{j}$ is nowhere dense in $B$ it cannot be a $\mathcal{G}_{\delta}$-set, and also the above proof would fail for this $B$. Thus $B$ cannot be a union of perfect sets, unless another proof is used. 
For $g$ strictly increasing in $[a, b]$ we now define an integral $(N) \int_{a}^{b} f d g$ using

$$
\begin{aligned}
& N_{+} D(F ; g ; u) \equiv \\
& \lim _{v \rightarrow u+}(S) \int_{u}^{v}(F(t)-F(u)) d_{t} N_{+}(u, v, t) /(S) \int_{u}^{v}(g(t)-g(u)) d_{t} N_{+}(u, v, t),
\end{aligned}
$$

$N_{-} D(F ; g ; v) \equiv$

$$
\lim _{u \rightarrow v^{-}}(S) \int_{u}^{v}(F(v)-F(t)) d_{t} N_{-}(u, v, t) /(S) \int_{u}^{v}(g(v)-g(t)) d_{t} N_{-}(u, v, t),
$$

in place of $(3,4)$, respectively. Upper and lower derivates follow on writing lim sup and lim inf, respectively, for the lim. A reader disliking Stieltjes integration can use $(3,4)$ instead, but the more general Stieltjes form allows us to use singular measures, and counting measures that give sums. Extending [23] we use functions $f, M$ from $[a, b]$ to R. $M$ is an $N_{s}$-Perron major function of $f, g$ on $[a, b]$, if and only if

$$
\begin{gathered}
M(a)=0 \text { and } M \text { is } N_{s} \text {-continuous, that is } \\
\lim _{v \rightarrow u^{+}}(S) \int_{u}^{v} M(t) d_{t} N_{+}(u, v, t)=M(u) \quad(a \leqslant u<b), \\
\lim _{u \rightarrow v^{-}}(S) \int_{u}^{v} M(t) d_{t} N_{-}(u, v, t)=M(v) \quad(a<v \leqslant b) ;
\end{gathered}
$$

$$
\text { the lower derivates } N_{+} \underline{D}(M ; g ; x) \geqslant f(x), N_{-} \underline{D}(M ; g ; x) \geqslant
$$
$f(x)$ g-almost everywhere on $[a, b]$;

the lower derivates are $-\infty$ only on a countable set of points of continuity of $\mathrm{g}$.

The infimum of $M(b)$ for all $N_{s}$-Perron major functions $M$ of $f, g$ on $[a, b]$ is called the upper $N_{s}$-Perron integral of $f, g$ on $[a, b]$. An $\mathrm{m}:[a, b] \rightarrow \mathbf{R}$ is an $N_{s}$-Perron minor function of $f, g$ on $[a, b]$ if and only if $-m$ is an $N_{s}$-Perron major function of $-f, g$ on $[a, b]$, and the lower $N_{s}$-Perron integral of $f, g$ on $[a, b]$, is the supremum of $m(b)$ for all such $m$. An $M:[a, b] \rightarrow \mathbf{R}$ is a strong $N_{s}$-Perron major function of $f, g$ on $[a, b]$, if and only if

$$
\begin{array}{ll}
N_{+} \underline{D}(M ; g ; t)>f(t) & (a \leqslant t<b), \\
N_{-} \underline{D}(M ; g ; t)>f(t) & (a<t \leqslant b), \quad M(a)=0,
\end{array}
$$

and $M$ is $N_{s}$-continuous, while strong $N_{s}$-Perron minor functions are defined using $-f$ as before. 
THEOREM 5. Let there be $a \delta(u)>0, a j(u)$ in $0<j(u)<1$ and depending only on $u$, and $k_{+}(u, h), x_{+}(u, h)$, continuous and monotone increasing and inverse functions in $h$, such that $0<k_{+}(u, h)<h$ and

$$
\begin{aligned}
N_{+}(u, u+h, u+h)-N_{+}\left(u, u+h, u+k_{+}(u, h)\right) & \geqslant j(u) \\
(0<h & \leqslant \delta(u), a \leqslant u<u+\delta(u) \leqslant b),
\end{aligned}
$$

and for each integrable $M$,

$$
j(u) L_{+}(u, h) \equiv \sup _{0<H \leqslant h}\left|(S) \int_{u}^{u+H}\{M(t)-M(u)\} d_{t} N_{+}(u, u+H, t)\right|
$$

is continuous in $h$ for $0<h \leqslant \delta(u), a \leqslant u<u+\delta(u) \leqslant b$. Similarly for $s=-$ in $(37,38)$. Then the upper and lower $N_{s}$-Perron integrals of $f, g$ on $[a, b]$ are unaltered if the major and minor functions are replaced by strong major and minor functions of the appropriate kinds, and also if $f$ is replaced by $f^{*}:[a, b] \rightarrow \mathbf{R}$ equal to $f, g$-almost everywhere.

Proof. As in Theorem 2 we first remove the finite or infinite sequence $\left(s_{n}\right)$ of exceptional points from (35) using the monotonicity of $N_{s}$ in $t$ and $L_{+}(u, h)$, $x_{+}(u, h)$ in $h$ so that the integrals in (39) below exist, and using (37), to get

$$
\begin{aligned}
(S) \int_{u}^{u+h} L_{+} & \left(u, x_{+}(u, t-u)\right) d_{t} N_{+}(u, v, t) \\
& \geqslant(S) \int_{u+k_{+}(u, h)}^{u+h} L_{+}\left(u, x_{+}\left(u, k_{+}(u, h)\right)\right) d_{t} N_{+}(u, v, t) \\
& \geqslant j(u) L_{+}(u, h) \\
& \geqslant\left|(S) \int_{u}^{u+h}\{M(t)-M(u)\} d_{t} N_{+}(u, u+h, t)\right| .
\end{aligned}
$$

As $M$ is $N_{s}$-continuous, given $\varepsilon>0$, there is an $H_{n}>0$ with

$$
j\left(s_{n}\right) L_{+}\left(s_{n}, H_{n}\right)<\varepsilon \cdot 2^{-n-1} \quad(n=1,2, \ldots) .
$$

Let

$$
N_{n+}\left(t+s_{n}\right)= \begin{cases}0 & (t \leqslant 0), \\ L_{+}\left(s_{n}, x_{+}\left(s_{n}, t\right)\right) & \left(0<t \leqslant k_{+}\left(s_{n}, H_{n}\right)\right), \\ L_{+}\left(s_{n}, H_{n}\right) & \left(t>k_{+}\left(s_{n}, H_{n}\right)\right),\end{cases}
$$

and similarly $N_{n-}(t)$ for $s=-$. Then by $(40,41)$,

$$
0 \leqslant \sum_{n=1}^{\infty}\left(N_{n+}(t)+N_{n-}(t)\right) \leqslant \varepsilon, \quad M_{1} \equiv M+\sum_{n=1}^{\infty}\left(N_{n+}+N_{n-}\right)
$$

is $N_{s}$-continuous as by $(33,38), L_{+}(u, h)$ is continuous in $h$, and

$$
N_{+} \underline{D}\left(M_{1} ; g ; s_{n}\right) \geqslant 0, \quad N_{-} \underline{D}\left(M_{1} ; g ; s_{n}\right) \geqslant 0 .
$$


As the $s_{n}$ are points of continuity of $g$ they form a set of $g$-measure zero, and so can be included in the exceptional set of (34) to give a set $X$ of $g$-measure zero, and so containing no jumps of $g$. By the construction of Theorem 2 with $g$-measure, we have a continuous $y(g ; t)$. Adding $y(g ; t)+\varepsilon g_{c}(t)$ to $M_{1}$, where $g_{c}$ is the continuous part of $g$, we have a strong $N_{s}$-Perron major function of $f, g$ in $[a, b]$ at most $\varepsilon(2+g(b))$ away from $M$, proving the theorem.

By (26), if $M, m$ are strong $N_{s}$-Perron major and minor functions, respectively, of $f, g$ in $[a, b]$ then $M(b) \geqslant m(b)$, and by Theorem 5 the same is true for $N_{s}$-Perron major and minor functions, and also the upper integral is not less than the lower integral, justifying the names. Further, the difference is monotone increasing, so that if the two are equal for $[a, b]$, with value called the $N_{s}$-Perron integral $P(b)$ of $f, g$ over $[a, b]$, they are equal for all $[a, t] \subseteq[a, b]$, while

$$
M(t) \geqslant P(t) \geqslant m(t)
$$

and $M-P$ and $P-m$ are monotone increasing.

If, given $f, g$, there is a $P:[a, b] \rightarrow \mathbf{R}$ with $P(a)=0$ and, given $\varepsilon>0$, there are a monotone increasing function $K(t)$ with $K(b)-K(a)<\varepsilon$ and a positive function $\delta(t)$ on $[a, b]$ such that for $a \leqslant u<v \leqslant b$ and respectively for $v<u+$ $\delta(u)$ and $u>v-\delta(v)$,

$$
\begin{aligned}
& (S) \int_{u}^{v}(K(t)-K(u)) d_{t} N_{+}(u, v, t) \\
& \quad \geqslant\left|(S) \int_{u}^{v}\{P(t)-P(u)-f(u)(g(t)-g(u))\} d_{t} N_{+}(u, v, t)\right|, \\
& (S) \int_{u}^{v}(K(v)-K(t)) d_{t} N_{-}(u, v, t) \\
& \quad \geqslant\left|(S) \int_{u}^{v}\{P(v)-P(t)-f(v)(g(v)-g(t))\} d_{t} N_{-}(u, v, t)\right|,
\end{aligned}
$$

we say that $P$ is the $N_{s}$-variational integral of $f, g$ over $[a, b]$.

THEOREM 6. If in Theorem 5, $f, g$ are $N_{s}$-Perron integrable to $P(t)$ over each $[a, t] \subseteq[a, b]$, then $P$, with $P(a)=0$, is the $N_{s}$-variational integral of $f, g$ over $[a, b]$; and conversely without using Theorem 5 .

Proof. If $f, g$ is $N_{s}$-Perron integrable to $P$, by Theorem 5 we can assume (36) true, and so

$$
\begin{aligned}
(S) \int_{u}^{v}(M(t)-M(u)) d_{t} N_{+}( & (u, v, t) \\
& \geqslant(S) \int_{u}^{v} f(u)(g(t)-g(u)) d_{t} N_{+}(u, v, t)
\end{aligned}
$$


for some $\delta_{1}(t)>0$ on $[a, b]$ and $a \leqslant u<v \leqslant b, v<u+\delta_{1}(u)$. By (42), $M=P$ $+K_{1}$ where $K_{1}$ is monotone increasing with

$$
\begin{aligned}
(S) \int_{u}^{v}\left(K_{1}(t)\right. & \left.-K_{1}(u)\right) d_{t} N_{+}(u, v, t) \\
& \geqslant(S) \int_{u}^{v}\{f(u)(g(t)-g(u))-P(t)+P(u)\} d_{t} N_{+}(u, v, t) .
\end{aligned}
$$

Similarly, for a strong $N_{s}$-Perron minor function $m$ we have a monotone increasing $K_{2}$ and a $\delta_{2}(t)>0$, with

$$
\begin{aligned}
(S) \int_{u}^{v}\left(K_{2}(t)\right. & \left.-K_{2}(u)\right) d_{t} N_{+}(u, v, t) \\
& \geqslant(S) \int_{u}^{v}\{P(t)-P(u)-f(u)(g(t)-g(u))\} d_{t} N_{+}(u, v, t)
\end{aligned}
$$

for $a \leqslant u<v \leqslant b, v<u+\delta_{2}(u)$. Taking $\delta(t) \equiv \min \left(\delta_{1}(t), \delta_{2}(t)\right)>0$ and $K=$ $K_{1}+K_{2}$, by choice of $M, m, K$ is monotone increasing with $K(b)-K(a)$ as small as we please. The same $K$ can be used for similar inequalities when $s=-$, so that $P$ is the $N_{s}$-variational integral.

Conversely, if $P$ is the $N_{s}$-variational integral then by $(43,44), P+K$ and $P-K$ are $N_{s}$-Perron major and minor functions, respectively, and $2 K(b)-2 K(a)$ is as small as we please. Hence $P$ is the $N_{s}$-Perron integral of $f, g$.

For an analogue of Theorem 1 we define two-dimensional integrals in $T \equiv$ $[a, b] \times[\alpha, \beta]$ using $N_{s}$ and $N_{\sigma}(s, \sigma=+$ or -$), N_{s}$ satisfying $(24,26)$ and the remarks involving (25), and $N_{\sigma}$ satisfying similar conditions with $\alpha, \beta, \sigma$ replacing $a, b, s$, respectively. If, given $f: T \rightarrow \mathbf{R}, g:[a, b] \rightarrow R, \gamma:[\alpha, \beta] \rightarrow \mathbf{R}$, there is a $P: T \rightarrow \mathbf{R}$ with $P(t, \tau)=0$ when $t=a$ or $\tau=\alpha$ or both, and, given $\varepsilon>0$, there are a $\delta(t, \tau)>0$ on $T$ and a $V: T \rightarrow \mathbf{R}$ with, for all such differences in $T$,

$$
\Delta^{2} V(a, b ; \alpha, \beta)<\varepsilon, \Delta^{2} V(u, t ; \mu, \tau) \geqslant 0,
$$

(S) $\int_{U} \Delta^{2} V(u, t ; \mu, \tau) d_{t, \tau} N_{s} N_{\sigma}$

$$
\begin{array}{r}
\geqslant\left|(S) \int_{U}\left\{\Delta^{2} P(u, t ; \mu, \tau)-f(u, \mu) \Delta_{1} g(u, t) \Delta_{2} \gamma(\mu, \tau)\right\} d_{t, \tau} N_{s} N_{\sigma}\right| \\
\left(U=[u, v] \times[\mu, \nu] \subseteq T,(v-u)^{2}+(\nu-\mu)^{2}<\delta^{2}(u, \mu), s=+=\sigma\right)
\end{array}
$$

and similar inequalities when $(+,+, u, \mu)$ are replaced by $(+,-, u, \nu)$, $(-,+, v, \mu),(-,-, v, \nu)$, we say that $P$ is the $N_{s} N_{\sigma}$-variational integral of $f, g \gamma$ over $T$.

THEOREM 7. Assuming that the S-integral is finitely additive in the integrand and obeys the conclusion of Theorem 7 for $S$, if $f, g$ are $N_{s}$-variationally integrable to 
$P(t)$ over each $[a, t] \subseteq[a, b]$, if $\varphi, \gamma$ are $N_{\mathrm{o}}$-variationally integrable to $\pi(\tau)$ over each $[\alpha, \tau] \subseteq[\alpha, \beta]$, and if $g, \gamma$ are of generalized bounded variation $\left(V B G^{*}\right)$ over their respective ranges, then $f \varphi, g \gamma$ are $N_{s} N_{\mathrm{o}}$-variationally integrable to $P(b) \pi(\beta)$ over $T$.

Proof. As in the proofs of A. J. Ward [49], page 581, Lemma 2, and page 592, Lemma 6, if $g, \gamma$ are VBG* there are $g_{j}, \gamma_{j}(j=1,2)$ such that $g_{1} \geqslant 0, \gamma_{1} \geqslant 0$, and $g_{2}, \gamma_{2}$ are finite and monotone increasing, and for small enough intervals,

$$
\left|\Delta_{1} g\right| \leqslant g_{1} \Delta_{1} g_{2}, \quad\left|\Delta_{2} \gamma\right| \leqslant \gamma_{1} \Delta_{2} \gamma_{2} \text {. }
$$

Given $\varepsilon>0$ and an integer $j>0$, there are $\delta_{j}(t)>0$ and monotone increasing $K_{j}(t)$ in $[a, b]$ such that $K_{j}(a)=0, K_{j}(b)<\varepsilon \cdot 4^{-j}$ and for $x=u$ or $v$ and $0<v-u<\delta_{j}(x)$,

$$
\begin{aligned}
(S) \int_{u}^{v}\left|K_{j}(t)-K_{j}(x)\right| d_{t} N_{s}(u, v, t) & \\
& \geqslant\left|(S) \int_{u}^{v}\{f(x)(g(t)-g(x))-P(t)+P(x)\} d_{t} N_{s}(u, v, t)\right| .
\end{aligned}
$$

Also there are $\delta_{j}^{*}(\tau)>0$ and $K_{j}(\tau)$ with similar properties concerning $\varphi, \gamma \pi, \alpha, \beta$. Using $K, \mathrm{~K}$ from (22), and $Z_{j}, \delta(t, \tau)$ as in the proof of Theorem 1, except that $f$ and $\varphi$ are replaced by $f g_{1}$ and $\varphi \gamma_{1}$, and the analogue of (23) with $g$ and $\gamma$, and Theorem 7 for the $S$-integral, then for $(x, \xi)$ in $Z_{j} \backslash Z_{j-1}$ and $U=[u, v] \times[\mu, \nu]$,

$$
\begin{aligned}
& f(x) \varphi(\xi) \Delta_{1} g \Delta_{2} \gamma-\Delta_{1} P \Delta_{2} \pi=\left(f(x) \Delta_{1} g-\Delta_{1} P\right) \varphi(\xi) \Delta_{2} \gamma \\
&+ f(x) \Delta_{1} g\left(\varphi(\xi) \Delta_{2} \gamma-\Delta_{2} \pi\right)+\left(\Delta_{1} P-f(x) \Delta_{1} g\right)\left(\varphi(\xi) \Delta_{2} \gamma-\Delta_{2} \pi\right), \\
&\left|(S) \int_{U}\left\{f(x) \varphi(\xi) \Delta_{1} g \Delta_{2} \gamma-\Delta_{1} P \Delta_{2} \pi\right\} d_{t, \tau} N_{s} N_{\sigma}\right| \\
& \leqslant\left|(S) \int_{u}^{v}\left(f(x) \Delta_{1} g-\Delta_{1} P\right) d_{t} N_{s}\right||\varphi(\xi)| \cdot\left|(S) \int_{\mu}^{\nu} \Delta_{2} \gamma d_{\tau} N_{\sigma}\right| \\
&+|f(x)|\left|(S) \int_{u}^{v} \Delta_{1} g d_{t} N_{s}\right|\left|(S) \int_{\mu}^{\nu}\left\{\varphi(\xi) \Delta_{2} \gamma-\Delta_{2} \pi\right\} d_{\tau} N_{\sigma}\right| \\
&+\left|(S) \int_{u}^{v}\left(\Delta_{1} P-f(x) \Delta_{1} g\right) d_{t} N_{s}\right|\left|(S) \int_{\mu}^{\nu}\left(\varphi(\xi) \Delta_{2} \gamma-\Delta_{2} \pi\right) d_{\tau} N_{\sigma}\right| \\
& \leqslant(S) \int_{u}^{v} \Delta_{1} K_{j} d_{t} N_{s} \cdot 2^{j}(S) \int_{\mu}^{\nu} \Delta_{2} \gamma_{2} d_{\tau} N_{\sigma} \\
&+2^{j}(S) \int_{u}^{v} \Delta_{1} g_{2} d_{t} N_{s} \cdot(S) \int_{\mu}^{\nu} \Delta_{2} \mathrm{~K}_{j} d_{\tau} N_{\sigma} \\
&+(S) \int_{u}^{v} \Delta_{1} K_{j} d_{t} N_{s} \cdot(S) \int_{\mu}^{\nu} \Delta_{2} \mathrm{~K}_{j} d_{\tau} N_{\sigma} \leqslant(S) \int_{U} \Delta^{2} Q d_{d, \tau} N_{s} N_{\sigma}
\end{aligned}
$$


using (46) and

$$
\begin{aligned}
Q(t, \tau) & \equiv K(t) \gamma_{2}(\tau)+g_{2}(t) \mathrm{K}(\tau)+K(t) \mathrm{K}(\tau) \\
& <\varepsilon \gamma_{2}(\beta)+g_{2}(b) \varepsilon+\varepsilon^{2} \rightarrow 0
\end{aligned}
$$

as $\varepsilon \rightarrow 0+$ while $\Delta^{2} Q \geqslant 0$ and is finitely additive. Hence the proof,

A similar pattern of definitions and proofs holds for the $T$-integral or $M Z$-integral of J. Marcinkiewicz and A. Zygmund [34] and the $S C P$-integral of J. C. Burkill [10]. The result of J. J. McGrotty [35] is useful for integrals over $[a, b]$ but not for integrals over $[u, v] \subset[a, b]$, and a deeper theory would be necessary to deal with generalized Riemann integrals over subintervals of $[a, b]$. For the $S C P$-integral [10], pages 47-48, we use the Perron integral

$$
C(M ; u, v) \equiv \int_{u}^{v} M(t) d t /(v-u) .
$$

$M$ is $C$-continuous at $u$ if $C(M ; u, u+h) \rightarrow M(u)$ as $h \rightarrow 0$, and $M$ is $S C$-continuous at $u$ if

$$
\begin{aligned}
A(M ; u, h) & \equiv C(M ; u, u+h)-C(M ; u-h, u) \\
& =\int_{u}^{u+h}\{M(t)-M(u)\} d t / h-\int_{u-h}^{u}\{M(t)-M(u)\} d t / h \rightarrow 0
\end{aligned}
$$

with $h$. Here, $A(M ; u, h) / h$ is the ratio in (5) for this case, the upper and lower limits of which give the upper and lower $S C$-derivates. An $S C P$-major function $M$ of $f$ in $[a, b]$ involves a $B \subseteq[a, b], B$ containing $a, b$ and lying almost everywhere in $[a, b]$, such that $M$ is $C$-continuous in $B, S C$-continuous in $(a, b)$, and follows the analogues of $(34,35)$ with the given symmetric derivates. The analogue of Theorem 5 follows on dropping the \pm and taking $j(u), L(u, H), k(u, h), x(u, h)$ as, respectively,

$$
\frac{1}{2}, \sup _{0<h \leqslant H} 2|A(M ; u, h)|, \quad \frac{1}{2} h, \quad 2 h .
$$

For then $A\left(M_{1} ; s_{n}, h\right) \geqslant 0$ and the constructed $M_{1}-M$ is monotone increasing and continuous and so $S C$-continuous and $C$-continuous. Next, the $S C P$-integral $P(t)$ over [ $a, t]$ exists for $t$ in a suitable set $B$, see [10], pages 48, 49. The $S$-integral here is the Perron integral, and as $M$ is Perron integrable and $M-P$ is monotone increasing, $P$ must be Perron integrable. Thus the first part of the analogue of Theorem 6 goes through. So does the second part if we note that here the $N$-variational integral $P$ can be changed in a set of measure zero without falsifying the definition, and to make the definition exact we can suppose that $P$ is $C$-continuous whenever $C(P ; u, u+h)$ has a limit as $h \rightarrow 0$, that is, almost everywhere. Then the $N$-variational integral is the $S C P$-integral and the analogue of Theorem 7 is true. 
For the $M Z$-integral [34], pages 36-37 there is the added complication of the convergence or divergence of the Perron integral (taken a Lebesgue integral in most of [34]),

$$
\begin{aligned}
E(M ; u, h) & \equiv \lim _{\varepsilon \rightarrow 0+} \int_{\varepsilon \leqslant|u-t| \leqslant h}\{M(t)-M(u)\} /(2 t) d t \\
& =\lim _{\varepsilon \rightarrow 0+} \int_{\varepsilon}^{h} \frac{M(u+t)-M(u-t)}{2 t} d t
\end{aligned}
$$

Let $M:[a, b] \rightarrow R$ be Perron integrable and $C$-continuous in a suitable set $B$, and at a point $u$ let $E(M ; u, h)$ exist or be divergent to $+\infty$. In the case of divergence we write $B_{s} M(u)=+\infty$. Otherwise the Borel derivative $B_{s} M(u)$ is the limit of $E(M ; u, h) / h$ as $h \rightarrow 0+$. As $M$ is $C$-continuous in $B$ it is $S C$-continuous there and we can use the $M_{1}-M$ of the $S C P$-integral, and [34], page 35, (101), and we have

$$
E\left(M_{1} ; s_{n}, h\right)=\left[\frac{1}{2}\left(M_{1} ; s_{n}, t\right)\right]_{0}^{h}+\int_{0}^{h} \frac{A\left(M_{1} ; s_{n}, t\right)}{2 t} d t \geqslant 0 .
$$

Putting $\left(s_{n}\right)$ into the exceptional set $X$ of measure zero (which is not mentioned in [34]) we have a function $M_{2}$ with the required properties, so that the analogue of Theorem 5 is true. The $M Z$-integral is defined almost everywhere but not necessarily everywhere [34], page 38 , Section 46 , but as for the $S C P$-integral the analogues of Theorems 6,7 go through.

It may be that a different theory will be necessary to prove the analogue of Theorem 7 for (say) the integrals of James or Taylor, and I leave this problem to those interested.

\section{References}

[1] H. Bauer, 'Der Perronsche Integralbegriff und seine Beziehung zum Lebesgueschen', Monatsh. Math. Phys. 26 (1915), 153-198.

[2] L. S. Bosanquet, 'A property of Çesàro-Perron integrals', Proc. Edinburgh Math. Soc. (2) 6 (1940), 160-165, MR 2, 131.

[3] P. S. Bullen, 'The $P^{n}$-integral', J. Austral. Math. Soc. 14 (1972), 219-236, MR 47, 8783.

[4] P. S. Bullen and C. M. Lee, 'On the integrals of Perron type', Trans. Amer. Math. Soc. 182 (1973), 481-501, MR 49, 3057.

[5] P. S. Bullen and C. M. Lee, 'The $S C_{n} P$-integral and the $P^{n+1}$-integral', Canad. J. Math. 25 (1973), 1274-1284, MR 53, 13489.

[6] J. C. Burkill, 'The approximately continuous Perron integral', Math. Z. 34 (1931), 270-278, Zbl 2.386.

[7] J. C. Burkill, 'The Çesàro-Perron integral', Proc. London Math. Soc. (2) 34 (1932), 314-322, Zbl 5.392.

[8] J. C. Burkill, 'The Çesàro-Perron scale of integration', Proc. London Math. Soc. (2) 39 (1935), 541-552, Zbl 12.204. 
[9] J. C. Burkill, 'Fractional orders of integrability', J. London Math. Soc. 11 (1936), 220-226, Zbl 14.258 .

[10] J. C. Burkill, 'Integrals and trigonometric series', Proc. London Math. Soc. (3) 1 (1951), 46-57, MR 13, 126.

[11] G. E. Cross, 'The relation between two definite integrals', Proc. Amer. Math. Soc. 11 (1960), 578-579, MR 22, 8094.

[12] G. E. Cross, 'The relation between two symmetric integrals', Proc. Amer. Math. Soc. 14 (1963), 185-190, MR 26, 281.

[13] G. E. Cross, 'On the generality of the AP-integral', Canad. J. Math. 23 (1971), 557-561, MR 44, 378.

[14] G. E. Cross, 'The $P^{n}$-integral', Canad. Math. Bull. 18 (1975), 493-497, MR 53, 3224.

[15] G. E. Cross, 'Additivity of the $P^{n}$-integral', Canad. J. Math. 30 (1978), 783-796, MR 58, 6108.

[16] H. W. Ellis, 'Mean-continuous integrals', Canad. J. Math. 1 (1949), 113-124, MR 10, 520.

[17] S. Foglio, 'Absolute N-integration', J. London Math. Soc. 38 (1963), 87-88, MR 26, 3868.

[18] S. Foglio and R. Henstock, 'The $N$-variational integral and the Schwartz distributions-III', $J$. London Math. Soc. (2) 6 (1973), 693-700, MR 48, 2755.

[19] L. Gordon, 'Perron's integral for derivatives in $L^{r}$ ', Studia Math. 28 (1967), 295-316, MR 36, 322.

[20] H. Hake, 'Über de la Vallée Poussins Ober- und Unterfunktionen einfacher Integrale und die Integraldefinition von Perron', Math. Ann. 83 (1921), 119-142.

[21] R. Henstock, 'A new descriptive definition of the Ward integral', J. London Math. Soc. 35 (1960), 43-48, MR 22, 1648.

[22] R. Henstock, 'The use of convergence factors in Ward integration', Proc. London Math. Soc. (3) 10 (1960), 107-121, MR 22, 12197.

[23] R. Henstock, 'The equivalence of generalized forms of the Ward, variational, Denjoy-Stieltjes, and Perron-Stieltjes integrals', Proc. London Math. Soc. (3) 10 (1960), 281-303, MR 22, 12198.

[24] R. Henstock, ' $N$-variation and $N$-variational integrals of set functions', Proc. London Math. Soc. (3) 11 (1961), 109-133, MR 23 A995.

[25] R. Henstock, 'Definitions of Riemann type of the variational integrals', Proc. London Math. Soc. (3) 11 (1961), 402-418, MR 24, A1994.

[26] R. Henstock, 'Majorants in variational integration', Canad. J. Math. 18 (1966), 49-74, MR 32, 2545.

[27] R. Henstock, Linear analysis (Butterworths, London, 1968), MR 54, 7725.

[28] R. Henstock, 'Integration, variation and differentiation in division spaces', Proc. Roy. Irish Acad. Sect. A 78 (1978), 69-85, MR 80d: 26011.

[29] R. Henstock, 'The variation on the real line', Proc. Roy. Irish Acad. Sect. A 79 (1979), 1-10, MR 81d: 26005.

[30] R. D. James, 'Generalized $n$th primitives', Trans. Amer. Math. Soc. 76 (1954), 149-176, MR 15,611 .

[31] R. D. James, 'Summable trigonometric series', Pacific J. Math. 6(1956), 99-110, MR 17, 1198.

[32] R. L. Jeffery and D. S. Miller, 'Convergence factors for generalized integrals', Duke Math. J. 12 (1945), 127-142, MR 6, 204.

[33] H. Looman, 'Uber die Perronsche Integraldefinition', Math. Ann. 93 (1925), 153-156.

[34] J. Marcinkiewicz and A. Zygmund, 'On the differentiability of functions and summability of trigonometrical series', Fund. Math. 26 (1936), 1-43, Zbl 14.111.

[35] J. J. McGrotty, 'A theorem on complete sets', J. London Math. Soc. 37 (1962), 338-340, MR 25, 4052.

[36] H. P. McKean, Jr., Stochastic integrals (Academic Press, New York, 1969), MR 40, 947.

[37] E. J. McShane, Integration (Princeton Math. Series 7, 1944, Princeton Univ. Press), MR 6, 43.

[38] S. N. Mukhopadhyay, 'On the regularity of the $P^{n}$-integral and its application to summable trigonometric series', Pacific J. Math. 55 (1974), 233-247, MR 51, 10546.

[39] O. Perron, 'Ueber den Integralbegriff', Sitzungsber. Heidelb. Akad. Wiss. Math.-Natur. KI. 16 (1914), 1-16. 
[40] W. F. Pfeffer, 'The Perron integral in topological spaces' (Russian), Časopis Pěst. Mat. 88 (1963), 322-348, MR 32, 2546.

[41] W. F. Pfeffer, 'A definition of the integral in topological spaces', Ċasopis Pèst. Mat. 89 (1964), I 129-147, II 257-277, MR 32, 1318.

[42] S. Saks, Theory of the integral (2nd English edition, Warsaw, 1937), Zbl 17.300.

[43] W. L. C. Sargent, 'On a sufficient condition for a function integrable in the Çesàro-Perron sense to be monotonic', Quart. J. Math. Oxford Ser. 12 (1941), 148-153, MR 3, 228.

[44] D. N. Sarkhel, 'A criterion for Perron integrability', Proc. Amer. Math. Soc. 71 (1978), 109-112, MR 58, 17006.

[45] S. J. Taylor, 'An integral of Perron's type defined with the help of trigonometric series', Quart. J. Math. Oxford Ser. (2) 6 (1955), 255-274, MR 19, 255.

[46] B. S. Thomson, 'Constructive definitions for non-absolutely convergent integrals', Proc. London Math. Soc. (3) 20 (1970), 699-716, MR 42, 3248.

[47] S. Verblunsky, 'On a descriptive definition of Çesàro-Perron integrals', J. London Math. Soc. (2) 3 (1971), 326-333, MR 44, 4161.

[48] S. Verblunsky, 'On the Peano derivatives', Proc. London Math. Soc. (3) 22 (1971), 313-324, MR 44, 2896.

[49] A. J. Ward, 'The Perron-Stieltjes integral', Math. Z. 41 (1936), 578-604, Zbl 14.397.

\section{School of Physical Sciences (Mathematics)}

The New University of Ulster

Coleraine

Northern Ireland 